$\mathrm{J}$ o u r n a l of

Mathematics

and Applications

JMA No 38, pp 171-180 (2015)

\title{
Application of the multi-step differential transform method to solve a fractional human T-cell lymphotropic virus I (HTLV-I) infection of CD4 ${ }^{+}$T-cells
}

\author{
Mohammad Zurigat, Mousa Ababneh
}

\begin{abstract}
Human T-cell Lymphotropic Virus I (HTLV-I) infection of $\mathrm{CD} 4^{+} \mathrm{T}$-Cells is one of the causes of health problems and continues to be one of the significant health challenges. In this article, a multi-step differential transform method is implemented to give approximate solutions of fractional modle of HTLV-I infection of CD4 ${ }^{+}$T-cells. Numerical results are compared to those obtained by the fourth-order Runge-Kutta method in the case of intger-order derivatives. The suggested method is efficient as the Runge-Kutta method. Some plots are presented to show the reliability and simplicity of the method.
\end{abstract}

AMS Subject Classification: 11 Y35, 65L05

Keywords and Phrases: Fractional differential equations; Multi-step differential transform method; Human T-cell Lymphotropic Virus Infection of CD4+ T-Cells; Numerical solution

\section{Introduction}

Human T-cell lymphotropic virus Type I (HTLV-I) infection is associated with member of the exogeneous human retroviruses that have a tropism for $\mathrm{T}$ lymphocytes. HTLV-I belongs to the delta-type retroviruses, which also include bovine leukemia virus; human T-cell leukemia virus Type II (HTLV-II), and simian T-cell leukemia virus. Human T-cell lymphotropic virus (HTLV) is a infection with HTLV-I is now a global epidemic, affecting 10 million to 20 million people. This virus has been linked to life-threatening, incurable diseases:

a) Adult T-cell leukemia (ATL).

b) HTLV-I-associated myelopathy/tropical spastic paraparesis.

COPYRIGHT (c) by Publishing Department Rzeszów University of Technology P.O. Box 85, 35-959 Rzeszów, Poland 
These syndromes are important causes of mortality and morbidity in the areas where HTLV-I is endemic, mainly in the tropics and subtropics. Mathematical models have proven valuable in understanding the dynamics of medical systems. Dynamic of HTLV-I infection of CD4 ${ }^{+}$T-cells is examined by $[1,2,3,4,5,6]$. The components of the basic four-component model are the concentration of healthy CD4 ${ }^{+} \mathrm{T}$-cells at time $\mathrm{t}$, the concentration of latently infected $\mathrm{CD} 4^{+} \mathrm{T}$-cells, the concentration of actively infected $\mathrm{CD}^{+}{ }^{+} \mathrm{T}$-cells and the concentration of leukemic cells at time t respectively, they are denoted by $T(t), I(t), V(t)$ and $L(t)$. These quantities satisfy:

$$
\begin{aligned}
\frac{d T(t)}{d t} & =\lambda-\mu_{T} T(t)-\kappa V(t) T(t), \\
\frac{d I(t)}{d t} & =\kappa_{1} V(t) T(t)-\left(\mu_{L}+\omega\right) I(t), \\
\frac{d V(t)}{d t} & =\omega I(t)-\left(\mu_{A}+\rho\right) V(t), \\
\frac{d L(t)}{d t} & =\rho V(t)+\beta L(t)\left(1-\frac{L(t)}{L_{\max }}\right)-\mu_{M} L(t) .
\end{aligned}
$$

With the initial conditions:

$$
T(0)=T_{0}, I(0)=I_{0}, V(0)=V_{0}, L(0)=L_{0} .
$$

The parameters $\lambda, \mu_{T}, \kappa$ and $\kappa_{1}$ are the source of CD4 ${ }^{+}$T-cells from precursors, the natural death rate of $\mathrm{CD} 4^{+} \mathrm{T}$-cells, the rate at which uninfected cells are contacted by actively infected cells, the rate of infection of T-cells with virus from actively infected cells, respectively. $\mu_{L}, \mu_{A}$ and $\mu_{M}$ are blanket death terms for latently infected, actively infected and leukemic cells. $\omega$ and $\rho$ represent the rates at which latently infected and actively infected cells become actively infected and leukemic, respectively. The rate $\beta$ determines the speed at which the saturation level for leukemia cells is reached. $T_{\max }$ is the maximal value that adult T-cell leukemia can reach. All parameters are assumed to be positive constants. In recent years, there has been a great deal of interest in fractional diffusion equations. These equations arise in viscous flows [7], biological models [8], evolution equations [9], reaction equations [10] and so on. Fractional derivatives provide an excellent instrument for the description of memory and hereditary properties of various materials and processes. Half-order derivatives and integrals prove to be more useful for the formulation of certain electrochemical problems than the classical models [11]. A great deal of effort has been expended over the last 10 years or so in attempting to find robust and stable numerical and analytical methods for solving fractional differential equations of physical interest. Our motivation for this work is to obtain the approximate solution of the fractional modle of HTLV-I infection of CD4 ${ }^{+}$T-Cells using the multi-step differential transform method (MSDTM). This method is only a simple modification of the differential transform method (DTM) $[12,13,14,15]$, in which it is treated as an algorithm in a sequence of small intervals (i.e. time step) for finding accurate approximate solutions to the corresponding systems. The approximate solutions obtained by using DTM are valid only for a short time. While the ones obtained by using the MSDTM [16] are more 
valid and accurate during a long time, and are in good agreement with the RK4-5 numerical solution when the order of the derivative $(\alpha=1)$. The rest of the paper is organized as follows. Section 2 gives an idea about the fractional calculus theory. In Section 3, we describe the MSDTM of the fractional order model of HTLV-I infection of $\mathrm{CD}^{+}{ }^{+}$T-Cells. Numerical simulations are presented graphically in Section 4. Finally, the conclusions are given in Section 5 .

\section{Fractional calculus}

Fractional calculus has been extensively applied in different fields. Many mathematicians and applied researchers have tried to model real processes using the fractional calculus. Jesus, Machado and Cunha [17] analyzed the fractional order dynamics in botanical electrical impedances. In biology, it has been deduced that the membranes of cells of biological organism have fractional order electrical conductance [18] and then are classified in groups of non-integer order models. Fractional order ordinary differential equations are naturally related to systems with memory which exists in most biological systems. Also, they are closely related to fractals, which are abundant in biological systems. We first give the definition of fractional-order integration and fractional-order differentiation $[19,20,21]$. There are several approaches to the generalization of the notion of differentiation to fractional orders e.g. Riemann-Liouville, Caputo and generalized functions approach. For the concept of fractional derivative, we will adopt Caputo's definition, which is a modification of the Riemann-Liouville definition and has the advantage of dealing properly with initial value problems.

Definition 2.1 A real function $f(x), \quad x>0$, is said to be in the space $C_{\alpha}, \alpha \in R$ if it can be written as $f(x)=x^{p} f_{1}(x)$, for some $p>\alpha$ where $f_{1}(x)$ is continous in $[0, \infty)$, and it is said to be in the space $C_{\alpha}^{m}$ if $f^{(m)} \in C_{\alpha}, m \in N$.

Definition 2.2 The fractional integral of order $\alpha>0$ of a function $f: R^{+} \longrightarrow R$ is given by

$$
\begin{aligned}
J^{\alpha} f(x) & =\frac{1}{\Gamma(\alpha)} \int_{0}^{x}(x-t)^{\alpha-1} f(t) d t, \quad \alpha>0, x>0, \\
J^{0} f(x) & =f(x) .
\end{aligned}
$$

Here we only need the following properties: For $f \in C_{\alpha}, \alpha, \beta>0, c \in R$ and $\gamma>-1$, we have

$$
\begin{aligned}
\left(J^{\alpha} J^{\beta}\right) f(x) & =J^{\alpha+\beta} f(x)=\left(J^{\beta} J^{\alpha}\right) f(x), \\
J^{\alpha} x^{\gamma} & =\frac{x^{\gamma+\alpha}}{\Gamma(\alpha)} B_{\tau}(\alpha, \gamma+1),
\end{aligned}
$$


where $B_{\tau}(\alpha, \gamma+1)$ is the incomplete beta function which is defined as

$$
B_{\tau}(\alpha, \gamma+1)=\int_{0}^{\tau} t^{\alpha-1}(1-t)^{\gamma} d t
$$

The Riemann-Liouville derivative has certain disadvantages when trying to model real world phenomena with fractional differential equations. Therefore, we shall introduce a modified fractional differential operator $D^{\alpha}$ proposed by Caputo in his work on the theory of viscoelasticity.

Definition 2.3 The Caputo fractional derivative of $f(x)$ of order $\alpha>0$ is defined as

$$
D^{\alpha} f(x)=\left(J^{m-\alpha}\right) f^{(m)}(x)=\frac{1}{\Gamma(m-\alpha)} \int_{0}^{x} \frac{f^{(m)}(t)}{(x-t)^{\alpha+1-m}} d t,
$$

for $m-1<\alpha \leq m, m \in N, x \geq 0, f(x) \in C_{-1}^{m}$. The Caputo fractional derivative was investigated by many authors, for $m-1<\alpha \leq m, f(x) \in C_{\alpha}^{m}$ and $\alpha \geq-1$, we have

$$
\left(J^{\alpha} D^{\alpha}\right) f(x)=J^{m} D^{m} f(x)=f(x)-\sum_{k=0}^{m-1} f^{(k)}(0) \frac{x^{k}}{k !} .
$$

For more mathematical properties of fractional derivatives and integrals one can back to the mentioned references.

\section{MSDTM Algorithm}

This paper attempts to find numerical solution for a general class of fractional order model of HTLV-I infection of CD4 ${ }^{+}$T-cells. Therefor, the paper summarizes specific techniques for MSDTM, as well as the applications of Caputo fractional calculus. The fractional order differential equations (FOD) are used becuace it are naturally related to systems with memory since the definition of fractional derivative involves an integration which is non local operator (as it is defined on an interval), so fractional derivative is a non local operator. Also, they are closely related to fractals which are abundant in biological systems. It has been shown that the approximated solutions obtained using DTM are not valid for large $t$ for some systems $[12,13,14,15]$. Therefore, we use the MSDTM to solve the following fractional order model of HTLV-I infection of $\mathrm{CD}^{+}{ }^{+}$T-Cells of order $0<\alpha \leq 1$ :

$$
\begin{aligned}
D^{\alpha} T(t) & =\lambda-\mu_{T} T(t)-\kappa V(t) T(t) \\
D^{\alpha} I(t) & =\kappa_{1} V(t) T(t)-\left(\mu_{L}+\omega\right) I(t), \\
D^{\alpha} V(t) & =\omega I(t)-\left(\mu_{A}+\rho\right) V(t) \\
D^{\alpha} L(t) & =\rho V(t)+\beta L(t)\left(1-\frac{L(t)}{L_{\max }}\right)-\mu_{M} L(t) .
\end{aligned}
$$


With the initial conditions:

$$
T(0)=1000, I(0)=250, V(0)=1.5, L(0)=0 .
$$

The method is a simple modification of the DTM, in which it is treated as an algorithm in a sequence of small intervals (i.e. time step) for finding accurate approximate solutions to the corresponding systems (8). This MSDTM offers accurate solutions over a longer time frame (more stable) compared to the standard DTM. Using the theorems given in [12] and taking the differential transform for the system (8) with respect to time t gives

$$
\begin{aligned}
T^{*}(k+1) & =\frac{\Gamma(\alpha k+1)}{\Gamma(\alpha(k+1)+1)}\left[\lambda \delta(k)-\mu_{T} T^{*}(k)-\kappa \sum_{l=0}^{k} V^{*}(l) T^{*}(k-l)\right] \\
I^{*}(k+1) & =\frac{\Gamma(\alpha k+1)}{\Gamma(\alpha(k+1)+1)}\left[\kappa_{1} \sum_{l=0}^{k} V^{*}(l) T^{*}(k-l)-\left(\mu_{L}+\omega\right) I^{*}(k)\right] \\
V^{*}(k+1) & =\frac{\Gamma(\alpha k+1)}{\Gamma(\alpha(k+1)+1)}\left[\omega I^{*}(k)-\left(\mu_{A}+\rho\right) V^{*}(k)\right] \\
L^{*}(k+1) & =\frac{\Gamma(\alpha k+1)}{\Gamma(\alpha(k+1)+1)}\left[\rho V^{*}(k)+\beta L^{*}(k)-\frac{\beta}{L_{\max }} \sum_{l=0}^{k} L^{*}(l) L^{*}(k-l)-\mu_{M} L^{*}(k)\right] .
\end{aligned}
$$

where $T^{*}(k), I^{*}(k), \quad V^{*}(k)$ and $L^{*}(k)$ are the differential transformations of $T(t), I(t), V(t)$ and $L(t)$, respectively. The differential transform of the initial conditions are given by $T^{*}(0)=1000, I^{*}(0)=250, V^{*}(0)=1.5$ and $L^{*}(0)=0$. In view of the differential inverse transform, the differential transform series solution for System (8) can be obtained as

$$
\begin{aligned}
T(t) & =\sum_{n=0}^{N} T^{*}(n) t^{\alpha n}, I(t)=\sum_{n=0}^{N} I^{*}(n) t^{\alpha n}, \\
V(t) & =\sum_{n=0}^{N} V^{*}(n) t^{\alpha n}, L(t)=\sum_{n=0}^{N} L^{*}(n) t^{\alpha n} .
\end{aligned}
$$

Now, according to the MSDTM, the series solution for the system (8) is suggested to be

$$
T(t)=\left\{\begin{array}{cc}
\sum_{n=0}^{K} T_{1}^{*}(n) t^{\alpha n}, & t \in\left[0, t_{1}\right], \\
\sum_{n=0}^{K} T_{2}^{*}(n)\left(t-t_{1}\right)^{\alpha n}, & t \in\left[t_{1}, t_{2}\right], \\
\vdots & \\
\sum_{n=0}^{K} T_{m}^{*}(n)\left(t-t_{m-1}\right)^{\alpha n}, & t \in\left[t_{m-1}, t_{m}\right],
\end{array}\right.
$$




$$
I(t)=\left\{\begin{array}{cc}
\sum_{n=0}^{K} I_{1}^{*}(n) t^{\alpha n}, & t \in\left[0, t_{1}\right], \\
\sum_{n=0}^{K} I_{2}^{*}(n)\left(t-t_{1}\right)^{\alpha n}, & t \in\left[t_{1}, t_{2}\right], \\
\vdots & t \in\left[t_{m-1}, t_{m}\right], \\
\sum_{n=0}^{K} I_{m}^{*}(n)\left(t-t_{m-1}\right)^{\alpha n}, & t \in\left[0, t_{1}\right], \\
\sum_{n=0}^{K} V_{1}^{*}(n) t^{\alpha n}, & t \in\left[t_{1}, t_{2}\right], \\
\sum_{n=0}^{K} V_{2}^{*}(n)\left(t-t_{1}\right)^{\alpha n}, & t \in\left[t_{m-1}, t_{m}\right], \\
\sum_{n=0}^{K} V_{m}^{*}(n)\left(t-t_{m-1}\right)^{\alpha n}, & t \in\left[t_{m-1}, t_{m}\right], \\
\sum_{n=0}^{K} L_{1}^{*}(n) t^{\alpha n}, & \left.t \in t_{1}, t_{2}\right], \\
\sum_{n=0}^{K} L_{2}^{*}(n)\left(t-t_{1}\right)^{\alpha n}, & \\
\sum_{n=0}^{K} L_{m}^{*}(n)\left(t-t_{m-1}\right)^{\alpha n}, &
\end{array}\right.
$$

where $T_{i}^{*}(n), I_{i}^{*}(n), V_{i}^{*}(n)$ and $L_{i}^{*}(n)$ for $i=1,2, \ldots, m$ satisfy the following recurrence relations

$$
\begin{aligned}
T_{i}^{*}(k+1) & =\frac{\Gamma(\alpha k+1)}{\Gamma(\alpha(k+1)+1)}\left[\lambda \delta(k)-\mu_{T} T_{i}^{*}(k)-\kappa \sum_{l=0}^{k} V_{i}^{*}(l) T_{i}^{*}(k-l)\right] \\
I_{i}^{*}(k+1) & =\frac{\Gamma(\alpha k+1)}{\Gamma(\alpha(k+1)+1)}\left[\kappa_{1} \sum_{l=0}^{k} V_{i}^{*}(l) T_{i}^{*}(k-l)-\left(\mu_{L}+\omega\right) I_{i}^{*}(k)\right] \\
V_{i}^{*}(k+1) & =\frac{\Gamma(\alpha k+1)}{\Gamma(\alpha(k+1)+1)}\left[\omega I_{i}^{*}(k)-\left(\mu_{A}+\rho\right) V_{i}^{*}(k)\right] \\
L_{i}^{*}(k+1) & =\frac{\Gamma(\alpha k+1)}{\Gamma(\alpha(k+1)+1)}\left[\rho V_{i}^{*}(k)+\beta L_{i}^{*}(k)-\frac{\beta}{L_{\max }} \sum_{l=0}^{k} L_{i}^{*}(l) L_{i}^{*}(k-l)-\mu_{M} L_{i}^{*}(k)\right]
\end{aligned}
$$

such that $T_{i}^{*}(0)=T_{i-1}^{*}(0), I_{i}^{*}(0)=I_{i-1}^{*}(0), V_{i}^{*}(0)=V_{i-1}^{*}(0)$ and $L_{i}^{*}(0)=L_{i-1}^{*}(0)$. Finally, if we start with $T_{o}^{*}(0)=1000, I_{0}^{*}(0)=250, V_{0}^{*}(0)=1.5$ and $L_{0}^{*}(0)=0$, using the recurrence relation given in System (16) then we can obtain the multi-step solution given in Systems (12)-(15). 

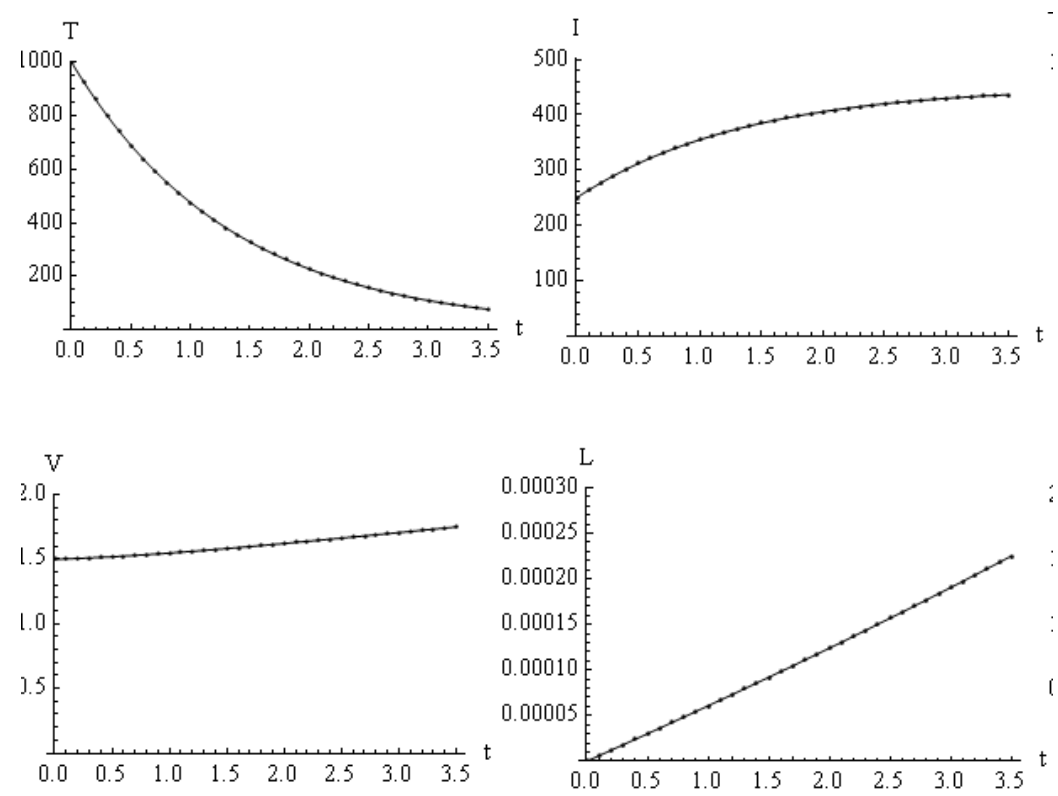

Figure 1: Plots of the components of lymphotropic virus I (HTLV) infection of CD4 ${ }^{+}$ T-cells model. Solid line: MSDTM solution, Dotted line: Runge-Kutta method solution

\section{Numerical results}

In this work, we propose the MSDTM, a reliable modification of the DTM that improves the convergence of the series solution. The method provides immediate and visible symbolic terms of analytic solutions, as well as numerical approximate solutions to both linear and nonlinear differential equations. Figure 1 shows the approximate solutions obtained using the MSDTM and the fourth-order Runge-Kutta method of the concentration of healthy $\mathrm{CD} 4^{+} \mathrm{T}$-cells at time $\mathrm{t}$, the concentration of latently infected $\mathrm{CD} 4^{+}$T-cells, the concentration of actively infected CD4 ${ }^{+} \mathrm{T}$-cells and the concentration of leukemic cells when $\alpha=1$ and the step size $\Delta t=0.1$. We assumed that all parameters are positive in $\mathrm{mm}^{3}$ /day as follows: $\lambda=6, \mu_{T}=$ $0.6, \mu_{L}=0.006, \mu_{A}=0.05, \mu_{M}=0.0005, \omega=0.0004, \rho=0.00004, \beta=0.0003$, $T_{\max }=2200$ and $\kappa=\kappa_{1}=0.1$. It can be seen that the results obtained based on MSDTM match the results of the Runge-Kutta method very well, which implies that the MSDTM can predict the behaviour of these variables accurately for the region under consideration. Next, interset to show how the concentrations of healthy CD4 ${ }^{+}$ T-cells, latently infected CD4 ${ }^{+}$T-cells, actively infected CD4 ${ }^{+}$T-cells and leukemic cells depend upon the magnitude of the order of fractional derivatives. We fix the parameters and perform a numerical simulation for different values of $\alpha$. Simulation results are presented in Figure 2. It it is clear that these solutions continuously depend 
on the fractional derivatives.
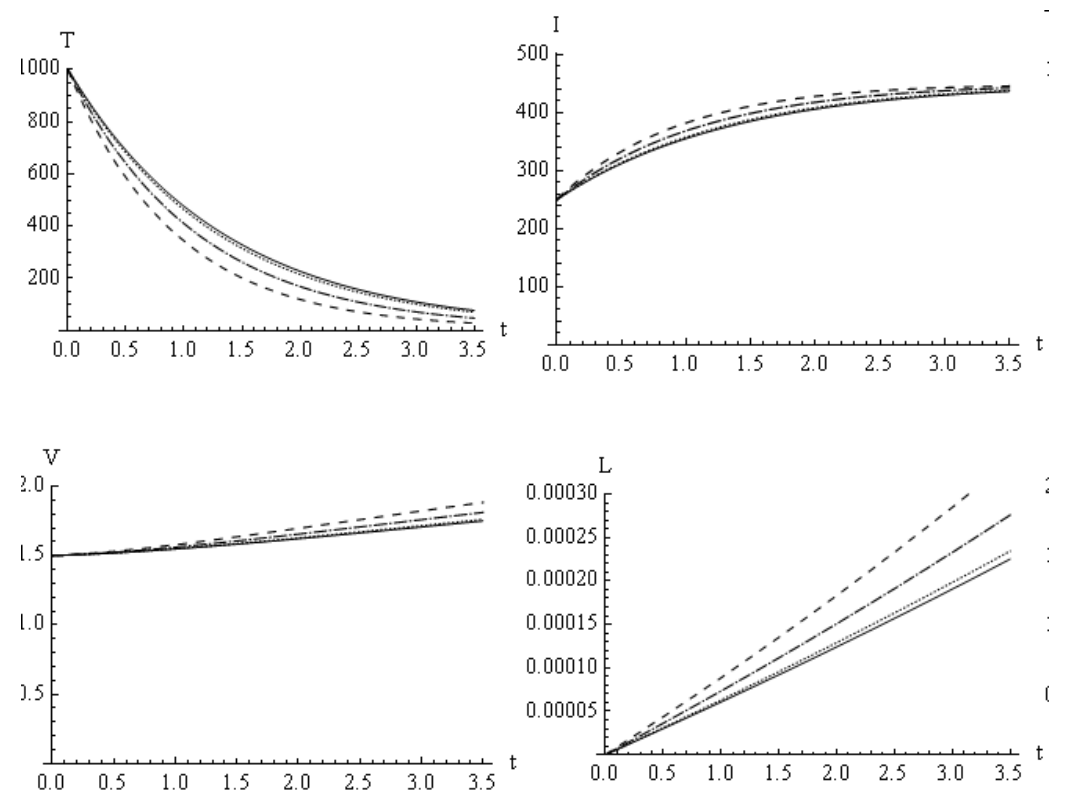

Figure 2: Plots of the components of lymphotropic virus I (HTLV) infection of CD4 ${ }^{+}$ T-cells model using MSDTM. Solid line: $(\alpha=1)$, Dotted line: $(\alpha=0.99)$, Dashed dotted line: $(\alpha=0.95)$, Dashed line: $(\alpha=0.9)$.

\section{Conclusions}

In this paper we employed the multi-step differential transform method in order to solve the fractional model of human T-cell lymphotropic virus I (HTLV-I) infection of $\mathrm{CD}^{+}$T-cells. Comparisons of the results obtained by using the MSDTM with that obtained by the classical Runge-Kutta method in the integer case reveal that the approximate solutions obtained by DTM are only valid for a small time, while the ones obtained by MSDTM are highly accurate and valid for a long time to nonlinear systems of differential equations. The reliability of the method and the reduction in the size of the computational domain give this method a wider applicability. It is of interest to note here that time fractional derivatives change the solutions, also we usually get in standard System (1). The concentration of healthy $\mathrm{CD} 4^{+}$T-cells $T(t)$, the concentration of latently infected $\mathrm{CD} 4^{+}$T-cells $I(T)$, the concentration of actively infected $\mathrm{CD} 4^{+}$T-cells $V(t)$ and the concentration of leukemic cells $L(t)$ have been obtained, therefore when $\alpha \longrightarrow 1$ the solution of the fractional model $(8) D^{\alpha} T(t), D^{\alpha} I(t), D^{\alpha} V(t), D^{\alpha} L(t)$ reduce to the standard solution $T(t), I(t), V(t), L(t)$ (see fig. 2). The recent appearance of nonlinear fractional 
differential equations as models in science and engineering makes it necessary to investigate the method of solutions for such equations. Consequently, the proposed method for the considered model verifes that it is a useful tool for these kind of models. The obtained results demonstrate the reliability of the algorithm and its wider applicability to fractional nonlinear evolution equations.

\section{References}

[1] N.I. Stilianakis, J. Seydel, Modeling the T-cell dynamics and pathogenesis of HTLV-I infection, Bull. Math. Biol. 61 (1999) 935-947.

[2] Patricia Katri, Shigui Ruan, Dynamics of human T-cell lymphotropic virus I (HTLV-I) infection of CD4+ T-cells, C. R. Biologies. 327 (2004) 1009-1016.

[3] L. Wang, M.Y. Li, D. Kirschner, Mathematical analysis of the global dynamics of a model for HTLV-I infection and ATL progression, Math. Biosci. 179 (2002) $207-217$.

[4] L.M. Petrovic, D.T. Spasic, T.M. Atanackovic, On a mathematical model of a human root dentin, Dental Materials. 21 (2005) 125_128.

[5] A. Gokdogan, M. Merdan, A multistage homotopy perturbation method for solving human T-cell lymphotropic virus I (HTLV-I) infection of CD4 ${ }^{+}$T-cells model, Middle-East Journal of Scientific Research. 9 (4) (2011) 503-509.

[6] X. Y. Shi, H. Gao, V. I. Lazouskaya, Q. Kang, Y. Jin, L.P. Wang, Viscous flow and colloid transport near air-water interface in a microchannel, Computers \& Mathematics with Applications. 59 (7) (2010) 2290-2304.

[7] A. Gokdogan, M. Merdan, A multistage homotopy perturbation method for solving human T-cell lymphotropic virus I (HTLV-I) infection of CD4 ${ }^{+}$T-cells model, Middle-East Journal of Scientific Research. 9 (4) (2011) 503-509.

[8] G. Barro, O. So, J. M. Ntaganda, B. Mampassi, B. Some, A numerical method for some nonlinear differential equation models in biology, Applied Mathematics and Computation. 200(1) (2008) 28-33.

[9] A. M. Wazwaz, New solutions of distinct physical structures to high-dimensional nonlinear evolution equations, Applied Mathematics and Computation. 196(1) (2008) 363-370.

[10] I. Hashim, M.S.H. Chowdhury, S. Mawa, On multistage homotopy-perturbation method applied to nonlinear biochemical reaction model, Chaos, Solitons \& Fractals. 36(4) (2008) 823-827.

[11] I. Podlubny, Fractional Differential Equations, Academic Press, New York, 1999. 
[12] Z. Odibat, S. Momani, V.S. Ertürk, Generalized differential transform method: Application to differential equations of fractional order, Appl. Math. Comput. 197 (2008) 467-477.

[13] S. Momani, Z. Odibat, A novel method for nonlinear fractional partial differential equations: Combination of DTM and generalized Taylor's formula, Journal of Computational and Applied Mathematics. 220 (2008) 85-95.

[14] Z. Odibat, S. Momani, A generalized differential transform method for linear partial differential equations of fractional order, Applied Mathematics Letters. 21 (2008) 194-199.

[15] V.S. Ertürk, S. Momani, Z. Odibat, Application of generalized differential transform method to multi-order fractional differential equations, Communications in Nonlinear Science and Numerical Simulation. 13 (2008) 1642-1654.

[16] Z. Odibat, C. Bertelle, M.A. Aziz-Alaoui, G. Duchamp, A multi-step differential transform method and application to non-chaotic or chaotic systems, Computers \& Mathematics with Applications. 59(4) (2010) 1462-1472.

[17] I. S. Jesus, J. A. Machado, J. B. Cunha, Fractional electrical impedances in botanical elements, Journal of Vibration and Control. 14 (2008) 1389-1402.

[18] K. S. Cole, Electric conductance of biological systems, Proc Cold Spring Harbor Symp Quant. Biol, Cold Spring Harbor. New York (1993) 107-116.

[19] A.A. Kilbas, H.M. Srivastava, J.J. Trujillo, Theory and Applications of Fractional Differential Equations, Elsevier, Amsterdam, (2006).

[20] S. Das, Functional Fractional Calculus, Springer, (2011).

[21] R.L. Magin, Fractional Calculus in Bioengineering, Begell House Publishers, (2006).

DOI: $10.7862 / \mathrm{rf} .2015 .13$

Mohammad Zurigat - corresponding author

email: moh_zur@hotmail.com

Department of Mathematics, Al Al-Bayt University, P.O. Box: 130095 Mafraq, Jordan.

Mousa Ababneh

Department of Mathematics, Al-Balqa Applied University, Salt, Jordan.

Received 20.11.2013 